\title{
Correction to: Sperm miR-34c-5p Transcript Content and Its Association with Sperm Parameters in Unexplained Infertile Men
}

\author{
Mehran Dorostghoal $^{1,2} \cdot$ Hamid Galehdari $^{1} \cdot$ Masoud Hemadi $^{3} \cdot$ Elahe Davoodi $^{1}$
}

Published online: 29 October 2021

(c) Society for Reproductive Investigation 2021

\section{Correction to: Reprod. Sci.}

https://doi.org/10.1007/s43032-021-00733-w

This article was updated to correct a spelling error in the title.

Publisher's Note Springer Nature remains neutral with regard to jurisdictional claims in published maps and institutional affiliations.

The online version of the original article can be found at https:// doi.org/10.1007/s43032-021-00733-w

Mehran Dorostghoal

mdorostghoal@scu.ac.ir; dorostghoal@gmail.com

1 Department of Biology, Faculty of Science, Shahid Chamran University of Ahvaz, Ahvaz, Iran

2 Biotechnology and Bioscience Research Center, Shahid Chamran University of Ahvaz, Ahvaz, Iran

3 Obstetrics and Gynecology, Fertility, Infertility and Perinatology Research Center, Ahvaz Jundishapur University of Medical Science, Ahvaz, Iran 